\title{
GMR
}

\section{Dynamic comparison of genetic diversity in a Small Tail Han sheep population using meta-analysis}

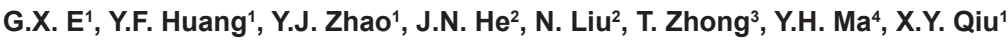 \\ and L.P. Chen ${ }^{1}$ \\ ${ }^{1}$ College of Animal Science and Technology, \\ Chongqing Key Laboratory of Forage \& Herbivore, \\ Chongqing Engineering Research Centre for Herbivores Resource Protection and \\ Utilization, Southwest University, Chongqing, China \\ ${ }^{2}$ College of Animal Science and Technology, Qingdao Agricultural University, \\ Qingdao, China \\ ${ }^{3}$ Farm Animal Genetic Resources Exploration and Innovation Key Laboratory of \\ Sichuan province, Sichuan Agricultural University, Chengdu, Sichuan, China \\ ${ }^{4}$ Institute of Animal Science, Chinese Academy of Agricultural Sciences, Beijing, China \\ Corresponding author: Y.F. Huang \\ E-mail: H67738337@swu.edu.cn
}

Genet. Mol. Res. 14 (4): 14607-14614 (2015)

Received June 23, 2015

Accepted September 2, 2015

Published November 18, 2015

DOI http://dx.doi.org/10.4238/2015.November.18.24

ABSTRACT. The aim of this research was to identify the dynamic diversity of Small Tail Han sheep in its main producing areas between different years, and provide a basis for a breeding and genetic resources conservation strategy. For this purpose, 15 microsatellites were genotyped for Small Tail Han Sheep sampled in 2014 from Heze, China, and a comparative analysis of these data with those from a previous study was undertaken using meta-analysis. The results reveal that inbreeding has caused a reduction in diversity of Small Tail Han Sheep from 2008 to 2014. Overall, 
our results are helpful in understanding the dynamic change in diversity, as well as providing information for a conservation strategy for this population.

Key words: Small Tail Han sheep; Diversity; Microsatellite; Inbreeding; Genetic resource conservation

\section{INTRODUCTION}

Sheep (Ovis aries) is a predominantly domestic animal, and it not only provides meat, but also milk, wool, and fur. Currently, a wide array of sheep breeds with abundant phenotypic diversity exists as a result of domestication and selection. However, commercial lines and industrialized livestock production systems have spread across continents, resulting in a decrease of large indigenous sheep populations in comparison to some commercial breeds. Therefore, it seems particularly important to build a complete monitoring system of conservation genetic resources for sheep. Small Tail Han sheep is an indigenous sheep genetic resource in the reaches of the Yellow River in China, where they live in an alpine climate (between 30 and $60 \mathrm{~m}$ ). It is a commercially important domestic species, characterized with a middle to large body size and preeminent fecundity (China National Commission of Animal Genetic Resources, 2011).

In the past, many studies have investigated functional candidate genes in Small Tail Han Sheep, such as during prolificacy, including bone morphogenetic protein 15 (BMP15; Chu et al., 2005), BMP4 (Chu et al., 2008), blood protein (Yang and Luo, 2004), gonadotropin releasing hormone receptor (GnRHR; Sun et al., 2008), polymorphism of serum esterase (Yang and Li, 2009), Booroola (FecB), and Inverdale (FecXI) mutations (Davis et al., 2006). Furthermore, the association of FecB (Chu et al., 2007), insulin-like factor1 gene (IGF1; He et al., 2012), follicle-stimulating hormone receptor (FSHR; Chu et al., 2012a), luteinizing hormone receptor (LHR; Jia et al., 2007), inhibin BB (Chu et al., 2011) and Kisspeptin-1 (KISS-1) and G protein coupled receptor 54 (GPR54; Chu et al., 2012b) with litter size have been investigated. To date, with the development of biotechnology, a large number of studies of Small Tail Han sheep have been undertaken using next generation sequencing technology, such as transcriptome analysis between Small Tail Han sheep and the Surabaya fur sheep (Miao and Luo, 2013), and comparative muscle transcriptome (Zhang et al., 2013) or miRNA (Miao et al., 2015) in Dorper and Small Tail Han sheep using high-throughput RNA sequencing.

With regard to the estimated diversity of sheep, recently, a series of reports were published worldwide (e.g., Arora et al., 2011; Paiva et al., 2011; Crispim et al., 2013; Ferreira et al., 2014; Yilmaz et al., 2014; Pons et al., 2015), and particularly in China (e.g., Sun et al., 2007; Zhong et al., 2011). However, most studies focused on diversity estimation and population structure and no study concentrated on dynamic change or group status. It is meaningless and less or without contribution to genetic protection strategy for indigenous sheep. The aim of this research was to identify the dynamic development of diversity and provide a basis for a breeding and genetic resources conservation strategy in Small Tail Han sheep in its main producing areas.

\section{MATERIAL AND METHODS}

\section{Sample collection and genotyping}

We genotyped 32 unrelated Small Tail Han sheep individuals, which were sampled from the concordant region as previously reported by Zhong et al. (2011) in its main producing area 
of Heze, China in September 2014. Individuals were genotyped at fifteen previously developed microsatellite loci (Kappes et al., 1997; Maddox et al., 2001; FAO, 2011), which have been suggested for biodiversity studies in sheep (Table 1). Approximately 1 to $2 \mu \mathrm{L}$ PCR product was diluted with $10 \mu \mathrm{L}$ of autoclaved distilled water for use in DNA genotyping. Diluted products $(2 \mu \mathrm{L})$ were added to $7.75 \mu \mathrm{L} \mathrm{Hi} \mathrm{Di \textrm {TM }}$ formamide and $0.25 \mu \mathrm{L}$ Gene Scan-500 $\mathrm{LIZ}^{\mathrm{TM}}$. The mixtures were heated at $94^{\circ} \mathrm{C}$ for $5 \mathrm{~min}$ and then immediately chilled on ice for $2 \mathrm{~min}$. Genotyping was performed on a Genetic Analyzer 3130 xl (AB Applied Biosystems).

Table 1. Microsatellite primers used to genotype Small Tail Han sheep.

\begin{tabular}{|c|c|c|}
\hline Name & Direction & Primer sequence $\left(5^{\prime}\right.$ to $\left.3^{\prime}\right)$ \\
\hline \multirow[t]{2}{*}{ HSC } & Forward & CTGCCAATGCAGAGACACAAGA \\
\hline & Reverse & GTCTGTCTCCTGTCTTGTCATC \\
\hline \multirow[t]{2}{*}{ McM42 } & Forward & CATCTTTCAAAAGAACTCCGAAAGTG \\
\hline & Reverse & CTTGGAATCCTTCCTAACTTTCGG \\
\hline \multirow[t]{2}{*}{ MCM527 } & Forward & GTCCATTGCCTCAAATCAATTC \\
\hline & Reverse & AAACCACTTGACTACTCCCCAA \\
\hline \multirow[t]{2}{*}{ OarAE129 } & Forward & AATCCAGTGTGTGAAAGACTAATCCAG \\
\hline & Reverse & GTAGATCAAGATATAGAATATTTTTCAACACC \\
\hline \multirow[t]{2}{*}{ OarFCB11 } & Forward & GCAAGCAGGTTCTTTACCACTAGCACC \\
\hline & Reverse & GGACTGAACTCACAAGTTGATATATCTATCAC \\
\hline \multirow[t]{2}{*}{ ILSTS005 } & Forward & GGAAGCAATGAAATCTATAGCC \\
\hline & Reverse & TGTTCTGTGAGTTTGTAAGC \\
\hline \multirow[t]{2}{*}{ OarFCB129 } & Forward & GCGACTTAGCAGCAGCAGCATCC \\
\hline & Reverse & CATCAAGAGATGAATGAGTAAAGAAGATG \\
\hline \multirow[t]{2}{*}{ OarFCB20 } & Forward & AAATGTGTTTAAGATTCCATACAGTG \\
\hline & Reverse & GGAAAACCCCCATATATACCTATAC \\
\hline \multirow[t]{2}{*}{ MAF209 } & Forward & GATCACAAAAAGTTGGATACAACCGTG \\
\hline & Reverse & TCATGCACTTAAGTATGTAGGATGCTG \\
\hline \multirow[t]{2}{*}{ OarFCB304 } & Forward & CCCTAGGAGCTTTCAATAAAGAAI,CGG \\
\hline & Reverse & CGCTGCTGTCAACTGGGTCAGGG \\
\hline \multirow[t]{2}{*}{ OarJMP29 } & Forward & GTATACACGTGGACACCGCTTTGTAC \\
\hline & Reverse & GAAGTGGCAAGATTCAGAGGGGAAG \\
\hline \multirow[t]{2}{*}{ ILSTS44 } & Forward & AGTCACCCAAAAGTAACTGG \\
\hline & Reverse & ACATGTTGTATTCCAAGTGC \\
\hline \multirow[t]{2}{*}{ MAF65 } & Forward & AAAGGCCAGAGTATGCAATTAGGAG \\
\hline & Reverse & ССАСТССТССTGAGAATATAACATG \\
\hline \multirow[t]{2}{*}{ ILSTS49 } & Forward & САATTTTCTTGTСTСTСССС \\
\hline & Reverse & GCTGAATCTTGTCAAACAGG \\
\hline \multirow[t]{2}{*}{ TGLA53 } & Forward & GCTTTCAGAAATAGTTTGCATTCA \\
\hline & Reverse & ATCTTCACATGATATTACAGCAGA \\
\hline
\end{tabular}

\section{Data from a previous study of Small Tail Han sheep}

The genotypes of 48 individuals of Small Tail Han sheep based on 19 microsatellite loci and collected in 2008 was offered by Dr. Zhong (Farm Animal Genetic Resources Exploration and Innovation Key Laboratory of Sichuan province, Sichuan Agricultural University, Chengdu, Sichuan 625014, China; Zhong et al., 2011).

\section{Meta dataset}

Of the microsatellite loci used in this study and the previous study (Zhong et al., 2011), there were six markers uniform to both studies (MCM527, ILSTS005, MAF209, OarJMP29, OarAE129, and OarFCB304). Twenty random samples from those collected in 2008 were re-genotyped with the 32 new individuals from 2014 using the six uniform microsatellites to provide insight on the consistency between the two datasets from 2008 and 2014. 


\section{Statistical and data analysis}

Expected $\left(H_{\mathrm{E}}\right)$ and observed $\left(H_{\mathrm{O}}\right)$ heterozygosity, mean number of alleles $\left(N_{\mathrm{A}}\right)$, and polymorphism information content (PIC) were estimated from the allele frequencies using the Microsatellite Toolkit (Park, 2001). For each locus-population combination of the global data set and population groupings, we used Fisher's exact test with Bonferroni correction to test for possible deviations from Hardy-Weinberg equilibrium (HWE) using GENEPOP 3.4 (Raymond and Rousset, 1995) and Arlequin software 3.5.1.3 (Excoffier and Lischer, 2010).

\section{RESULTS}

\section{Estimation of diversity in the 2014 Small Tail Han sheep}

In total, 217 alleles were found in the 2014 Small Tail Han sheep population across the 15 microsatellite loci. Across individuals, an average of 14.47 alleles per locus was observed, ranging from 9 in MCM527, ILSTS005, and MAF65 to 22 in ILSTS44.

Across individuals, mean $H_{\mathrm{O}}$ and $H_{\mathrm{E}}$ for all loci across the population were 0.603 (0.281 to 0.844 ) and 0.851 (0.751 to 0.927$)$, respectively (Table 2$)$. The average PIC across loci was 0.821 and ranged from 0.713 (ILSTS005) to 0.907 (OarFCB129). Across loci, $N_{\mathrm{A}}$ was $14.467 \pm 4.291, H_{\mathrm{O}}$ was $0.601 \pm 0.023$, and $H_{\mathrm{E}}$ was $0.851 \pm 0.016$.

Most loci in the 2014 Small Tail Han sheep population, except MCM527, deviated from HWE (Table 2). The inbreeding coefficient $\left(F_{\text {IS }}\right)$ for the 2014 population was also found to be significant $\left(F_{\mathrm{IS}}=0.295, \mathrm{P}=0.0033\right.$, Fisher's test $)$.

Table 2. Diversity and polymorphism information content (PIC) of the 2014 Small Tail Han sheep population based on fifteen microsatellite loci.

\begin{tabular}{|c|c|c|c|c|c|}
\hline Locus & $H_{0}$ & $H_{\mathrm{E}}$ & PIC & $F_{\text {IS }}$ & $\mathrm{P}$ value $\left(F_{\mathrm{IS}}\right)$ \\
\hline HSC\# & 0.3333 & 0.9271 & 0.9051 & 0.644 & $0.0033^{*}$ \\
\hline McM42 & 0.75 & 0.811 & 0.7727 & 0.076 & 0.2467 \\
\hline MCM527" & 0.5909 & 0.8594 & 0.8208 & 0.318 & 0.0100 \\
\hline OarAE129\# & 0.500 & 0.7881 & 0.7557 & 0.370 & $0.0033^{*}$ \\
\hline OarFCB11\# & 0.6875 & 0.9077 & 0.8846 & 0.246 & $0.0033^{*}$ \\
\hline ILSTS005\# & 0.3462 & 0.7511 & 0.7134 & 0.544 & $0.0033^{*}$ \\
\hline OarFCB129\# & 0.7813 & 0.9271 & 0.9066 & 0.159 & 0.0067 \\
\hline OarFCB20" & 0.7188 & 0.9112 & 0.8882 & 0.214 & 0.0133 \\
\hline MAF209" & 0.6452 & 0.7779 & 0.7335 & 0.173 & 0.0267 \\
\hline OarFCB304\# & 0.4516 & 0.825 & 0.799 & 0.457 & $0.0033^{*}$ \\
\hline OarJMP29\# & 0.75 & 0.8259 & 0.8015 & 0.093 & 0.0900 \\
\hline ILSTS44" & 0.7188 & 0.9142 & 0.8925 & 0.216 & $0.0033^{*}$ \\
\hline MAF65" & 0.2813 & 0.8041 & 0.7617 & 0.654 & $0.0033^{*}$ \\
\hline ILSTS49\# & 0.6452 & 0.8038 & 0.7707 & 0.200 & 0.0133 \\
\hline TGLA53 & 0.8438 & 0.9256 & 0.9049 & 0.090 & 0.0600 \\
\hline mean & 0.603 & 0.851 & 0.821 & 0.295 & $0.0033^{*}$ \\
\hline
\end{tabular}

Indicative adjusted nominal level (5\%) for the table is 0.00333 ; *denotes significant difference; ${ }^{*}$ indicates which loci deviated from Hardy-Weinberg equilibrium; $H_{\mathrm{O}}$, observed heterozygosity; $H_{\mathrm{E}}$, expected heterozygosity; $F_{\mathrm{IS}}$, inbreeding coefficient.

\section{Re-estimation of diversity in 2008 Small Tail Han sheep}

In total, 157 alleles were observed in the 2008 Small Tail Han sheep population across 15 microsatellite loci. Across individuals, an average of 8.26 alleles per locus was observed, ranging 
from 4 in SRCRSP5 to 16 in DYMS1.

Across individuals, mean $H_{\mathrm{O}}$ and $H_{\mathrm{E}}$ for all loci across the population were $0.664(0.381$ to 0.917 ) and 0.702 (0.489 to 0.866 ), respectively (Table 3). PIC across loci was 0.821 and ranged from 0.456 (OarCP38) to 0.842 (DYMS1). Across loci, $N_{\mathrm{A}}$ was $8.26 \pm 3.36, H_{\mathrm{O}}$ was $0.664 \pm 0.016$, and $H_{\mathrm{E}}$ was $0.702 \pm 0.026$. Only three loci deviated from HWE in the 2008 population, OarCP38, SRCRSP5, and OarAE129. $F_{\text {IS }}$ for the 2008 population was 0.054 and was not significant (Table 3).

\begin{tabular}{|c|c|c|c|c|c|}
\hline Locus & $H_{0}$ & $H_{\mathrm{E}}$ & $\mathrm{PIC}$ & $F_{\text {IS }}$ & $\mathrm{P}$ value $\left(F_{\mathrm{IS}}\right)$ \\
\hline OarCP38\# & 0.4167 & 0.490 & 0.4561 & 0.150 & 0.0737 \\
\hline SRCRSP9 & 0.6222 & 0.574 & 0.5224 & -0.085 & 0.8316 \\
\hline MAF214 & 0.8511 & 0.828 & 0.7975 & -0.028 & 0.7658 \\
\hline OarCP34 & 0.7708 & 0.745 & 0.6955 & -0.035 & 0.7211 \\
\hline OarVH72 & 0.6875 & 0.721 & 0.681 & 0.047 & 0.3000 \\
\hline OarFCB128 & 0.6667 & 0.757 & 0.7213 & 0.120 & 0.0421 \\
\hline OarHH47 & 0.8298 & 0.850 & 0.8249 & 0.024 & 0.4237 \\
\hline MCM527 & 0.6875 & 0.762 & 0.7162 & 0.099 & 0.1237 \\
\hline ILSTS5 & 0.5625 & 0.537 & 0.4972 & -0.049 & 0.7421 \\
\hline SRCRSP5 ${ }^{\#}$ & 0.381 & 0.605 & 0.5196 & 0.373 & 0.0079 \\
\hline MAF209 & 0.8947 & 0.8547 & 0.8257 & -0.047 & 0.8289 \\
\hline OarJMP29 & 0.6364 & 0.7074 & 0.6676 & 0.101 & 0.1421 \\
\hline OarFCB226 & 0.8511 & 0.7193 & 0.6678 & -0.186 & 0.9921 \\
\hline ILSTS28* & 0.5227 & 0.7827 & 0.7469 & 0.335 & $0.0026^{*}$ \\
\hline BM8125 & 0.4524 & 0.5364 & 0.4939 & 0.158 & 0.0974 \\
\hline DYMS1 & 0.9167 & 0.8658 & 0.8422 & -0.059 & 0.9211 \\
\hline ILSTS11 & 0.6250 & 0.6612 & 0.616 & 0.055 & 0.2868 \\
\hline OarAE129" & 0.4783 & 0.6061 & 0.5589 & 0.213 & 0.0237 \\
\hline OarFCB304 & 0.766 & 0.7282 & 0.7002 & -0.052 & 0.8605 \\
\hline mean & 0.664 & 0.702 & 0.661 & 0.054 & 0.0053 \\
\hline
\end{tabular}

Indicative adjusted nominal level (5\%) for one table is 0.00263 ; *denotes significant difference; \#indicates which loci deviated from Hardy-Weinberg equilibrium; $H_{\mathrm{O}}$, observed heterozygosity; $H_{\mathrm{E}}$, expected heterozygosity; $F_{\mathrm{IS}}$, inbreeding coefficient.

\section{Comparison of the dynamic change and group status of Small Tail Han sheep between 2008 and 2014 using six microsatellite loci}

In total, 49 and 50 alleles were found in the 2014 and 2008 Small Tail Han sheep populations across 6 microsatellite loci, respectively. For the 2014 population, an average of 8.2 alleles per locus was observed, ranging from 6 in OarAE129 to 10 in OarJMP29. For the 2008 population, an average of 8.3 alleles per locus was observed, ranging from 5 in OarAE129 to 14 in OarFCB304.

Across individuals, the mean $H_{\mathrm{O}}$ and $H_{\mathrm{E}}$ for all loci across the population was 0.651 ( 0.537 to 0.855$)$ and 0.669 ( 0.533 to 0.784 ) for the 2014 population and 0.671 ( 0.478 to 0.895 ) and 0.700 ( 0.537 to 0.855 ) for the 2008 population, respectively (Table 4 ). In addition, across loci, $N_{\mathrm{A}}=8.17 \pm 1.47, H_{\mathrm{O}}=0.651 \pm 0.034$, and $H_{\mathrm{E}}=0.700 \pm 0.038$ for the 2014 population, and $N_{\mathrm{A}}=8.33 \pm 3.27, H_{\mathrm{O}}=0.671 \pm 0.028$, and $H_{\mathrm{E}}=0.699 \pm 0.046$ for the 2008 population. $F_{\text {IS }}$ was 0.071 and 0.041 for the 2014 and 2008 populations, respectively, and was not significant (Table 5). 
Table 4. Diversity and polymorphism information content (PIC) of the 2008 and 2014 populations of Small Han sheep based on six uniform microsatellite loci.

\begin{tabular}{|c|c|c|c|c|c|c|}
\hline \multirow[t]{2}{*}{ Locus } & \multicolumn{2}{|c|}{$H_{\mathrm{E}}$} & \multicolumn{2}{|c|}{$H_{\mathrm{o}}$} & \multicolumn{2}{|c|}{ PIC } \\
\hline & 2014 & 2008 & 2014 & 2008 & 2014 & 2008 \\
\hline MCM527 & 0.765 & 0.762 & 0.813 & 0.688 & 0.730 & 0.716 \\
\hline ILSTS005 & 0.701 & 0.537 & 0.656 & 0.563 & 0.647 & 0.497 \\
\hline MAF209 & 0.754 & 0.855 & 0.688 & 0.895 & 0.704 & 0.826 \\
\hline OarJMP29 & 0.784 & 0.707 & 0.719 & 0.636 & 0.741 & 0.668 \\
\hline OarAE129 & 0.533 & 0.606 & 0.531 & 0.478 & 0.485 & 0.559 \\
\hline OarFCB304 & 0.664 & 0.728 & 0.500 & 0.766 & 0.616 & 0.700 \\
\hline
\end{tabular}

$H_{\mathrm{O}}$, observed heterozygosity; $H_{\mathrm{E}}$, expected heterozygosity.

Table 5. Comparative diversity between the 2008 and 2014 populations of Small Tail Han sheep based on six microsatellite loci.

\begin{tabular}{llcccccc}
\hline Sampled Year & Code & Sample Size & $H_{\mathrm{O}}$ & $H_{\mathrm{E}}$ & $N_{\mathrm{A}}$ & $F_{\text {IS }}$ & $P$ value $\left(F_{\text {IS }}\right)$ \\
\hline 2014 & SIH & 32 & $0.651 \pm 0.034$ & $0.700 \pm 0.038$ & $8.17 \pm 1.47$ & 0.071 & 0.0333 \\
2008 & SBW & 48 & $0.671 \pm 0.029$ & $0.699 \pm 0.046$ & $8.33 \pm 3.27$ & 0.041 & 0.0708 \\
\hline
\end{tabular}

Indicative adjusted nominal level (5\%) for the table is 0.00417 ; *denotes significant difference; \#indicates which loci deviated from Hardy-Weinberg equilibrium; $H_{\mathrm{O}}$, observed heterozygosity; $H_{\mathrm{E}}$, expected heterozygosity; $N_{\mathrm{A}}$, mean number of alleles; $F_{I S}$, inbreeding coefficient.

\section{DISCUSSION}

The majority of the results obtained in the current study are consistent with those from the previous study of Small Tail Han sheep undertaken in $2008\left(H_{\mathrm{O}}=0.661 \pm 0.014, H_{\mathrm{E}}=0.703\right.$ \pm 0.024 , and $N_{\mathrm{A}}=7.82 \pm 2.85$; Zhong et al., 2011). However, there are a number of differences. First, the 2008 population has a greater number of alleles than the 2014 population, and this is evident from looking at the results for each locus, e.g., OarFCB304 has 14 alleles in the 2008 population but only 8 alleles in the 2014 population. Second, only three loci (OarCP38, SRCRSP5, and OarAE129) deviated from HWE in the 2008 population, and there was only a small difference between $H_{\mathrm{E}}$ and $H_{\mathrm{O}}$ for each locus in this population, which means the Small Tail Han sheep in Heze, China was in a neutral and good risk status in 2008. However, in the Small Tail Han sheep population sampled in 2014, nearly all microsatellite loci deviated from HWE, except TGLA53. Thirdly, only one locus (ILSTS28) had a significant inbreeding coefficient in the 2008 population; however, in the 2014 population, more than half (8) of the microsatellite loci had significant and high $F_{\text {IS }}$ values. Finally, comparing the diversity in Small Tail Han sheep between 2008 and 2014 using six uniform loci revealed a smaller divergence between $H_{\mathrm{E}}$ and $H_{\mathrm{O}}$ in the 2008 population, and a larger difference between $H_{\mathrm{E}}$ and $H_{\mathrm{O}}$ in the 2014 population. These results indicate a decrease in diversity in the Small Tail Han sheep population from Heze between 2008 and 2014.

In short, from the comparative results of this study, it evident that inbreeding is reducing the diversity in the Small Tail Han sheep population at this moment in time. In addition, inbreeding results in homozygosity and this can increase the chances of offspring being affected by recessive or deleterious traits (Nabulsi et al., 2003). This generally leads to decreased biological fitness of a population (inbreeding depression) (Jiménez et al., 1994), which is its ability to survive and reproduce. Therefore, the conservation status of the genetic resources of the Small Tail Han sheep population from Heze is not optimistic. 


\section{Conflicts of interest}

The authors declare no conflict of interest.

\section{ACKNOWLEDGMENTS}

Research supported by the Fundamental Research Funds for the Central Universities (\#SWU114023) and the 2013 Innovation Team Building Program in Chongqing universities (\#KJTD201334). We thank Yuan-Zhi Sun and Chang-Hui Yu (Beijing Tianyi Huiyuan Bioscience \& Technology Inc, Beijing, 100070, China) for technical help.

\section{REFERENCES}

Arora R, Bhatia S, Mishra BP and Joshi BK (2011). Population structure in Indian sheep ascertained using microsatellite information. Anim. Genet. 42: 242-50.

China National Commission of Animal Genetic Resources (2011). Animal genetic resources in China: sheep and goats. Chinese Agricultural Press, Beijing.

Chu MX, Sang LH, Wang JY, Fang L, et al. (2005). Study on BMP15 and GDF9 as candidate genes for prolificacy of Small Tail Han sheep and Hu sheep. Acta Genetica Sin. 32: 38-45.

Chu MX, Liu ZH, Jiao CL, He YQ, et al. (2007). Mutations in BMPR-IB and BMP-15 genes are associated with litter size in Small Tailed Han sheep (Ovis aries). J. Anim. Sci. 85: 598-603.

Chu MX, Zhou WR, Sun SH, Fang L, et al. (2008). Polymorphism of BMP4 gene and its relationship with prolificacy of Small Tail Han sheep. J. Agric. Biotechnol. 16: 237-241.

Chu MX, Zhuang H, Zhang Y, Jin M, et al. (2011). Polymorphism of Inhibin $\beta B$ gene and its relationship with litter size in sheep. Anim. Sci. J. 82: 57-61.

Chu MX, Guo XH, Feng CJ, Li Y, et al. (2012a). Polymorphism of 5' regulatory region of ovine FSHR gene and its association with litter size in Small Tail Han sheep. Mol. Biol. Rep. 39: 3721-3725.

Chu MX, Xiao C, Feng T, Fu Y, et al. (2012b). Polymorphisms of KISS-1 and GPR54 genes and their relationships with litter size in sheep. Mol. Biol. Rep. 39: 3291-3297.

Crispim BA, Grisolia AB, Seno LO, Egito AA, et al. (2013). Genetic diversity of locally adapted sheep from Pantanal region of Mato Grosso do Sul. Genet. Mol. Res. 12: 5458-5466.

Davis GH, Balakrishnan L, Ross IK, Wilson T, et al. (2006). Investigation of the Booroola (FecB) and Inverdale (FecX(I)) mutations in 21 prolific breeds and strains of sheep sampled in 13 countries. Anim. Reprod. Sci. 92: 87-96.

Excoffier L and Lischer HE (2010). Arlequin suite ver 3.5: a new series of programs to perform population genetics analyses under Linux and Windows. Mol. Ecol. Resour. 10: 564-567.

Ferreira JS, Paiva SR, Silva EC, McManus CM, et al. (2014). Genetic diversity and population structure of different varieties of Morada Nova hair sheep from Brazil. Genet. Mol. Res. 13: 2480-90.

He JN, Zhang BY, Chu MX, Wang PQ, et al. (2012). Polymorphism of insulin-like growth factor 1 gene and its association with litter size in Small Tail Han sheep. Mol. Biol. Rep. 39: 9801-9807.

Jia CL, Ning LI, Wei ZH, Zhu XP, et al. (2007). Study on FSHR and LHR mRNA levels of different BMPRIB genotypes from Small Tail Han sheep during the oestrum. Agr. Sci. China 6: 94-99.

Jiménez JA, Hughes KA, Alaks G, Graham L, et al. (1994). An experimental study of inbreeding depression in a natural habitat. Science 266: 271-273.

Miao X and Luo Q (2013). Genome-wide transcriptome analysis between small-tail Han sheep and the Surabaya fur sheep using high-throughput RNA sequencing. Reproduction 145: 587-596.

Miao X, Luo Q and Qin X (2015). Genome-Wide analysis reveals the differential regulations of mRNAs and miRNAs in Dorset and Small Tail Han sheep muscles. Gene 562: 188-196.

Nabulsi MM, Tamim H, Sabbagh M, Obeid MY, et al. (2003). Parental consanguinity and congenital heart malformations in a developing country. Am. J. Med. Genet. A 116A: 342-347.

Paiva SR, Facó O, Faria DA, Lacerda T, et al. (2011). Molecular and pedigree analysis applied to conservation of animal genetic resources: the case of Brazilian Somali hair sheep. Trop. Anim. Health Prod. 43: 1449-1457.

Park SDE (2001). Trypanotolerance in West African cattle and the population genetic effects of selection. Doctoral thesis, University of Dublin, Dublin. 
Pons AL, Landi V, Martinez A and Delgado JV (2015). The biodiversity and genetic structure of Balearic sheep breeds. J. Anim. Breed. Genet. 132: 268-276.

Raymond $M$ and Rousset F (1995). GENEPOP (Version 1.2): population genetics software for exact tests and ecumenicism. J. Hered. 86: 248-249.

Sun J, Chu MX, Chen HQ, Fang L, et al. (2008). Polymorphism of GnRHR gene and its relationship with prolificacy of small tail Han sheep. J. Agric. Biotechnol. 16: 230-236.

Sun W, Chang H, Ji D, Liao X, et al. (2007). Analysis on genetic diversity and isolation mechanism by distance of different ecological type sheep breeds in Mongolia sheep group. J. Genet. Genomics 34: 1001-1009.

Yang CQ and Li XY (2009). Serum esterase polymorphisms and their associations with prolificacy in Small Tail Han sheep. Anim. Husbandry and Feed Sci. 1: 9-11.

Yang GL and Luo YZ (2004). Correlation of blood protein polymorphism to prolificacy of Small-tail han sheep. J. Gansu Agr. Univ. 39: 381-385.

Yilmaz O, Cemal I and Karaca O (2014). Genetic diversity in nine native Turkish sheep breeds based on microsatellite analysis. Anim. Genet. 45: 604-608.

Zhang C, Wang G, Wang J, Ji Z, et al. (2013). Characterization and comparative analyses of muscle transcriptome in Dorper and Small-Tailed Han sheep using RNA-seq technique. PLoS One 8: e72686.

Zhong T, Han JL, Guo J, Zhao QJ, et al. (2011). Tracing genetic differentiation of Chinese Mongolian sheep using microsatellites. Anim. Genet. 42: 563-565. 